\title{
Modern facilities for experimental measurement of dynamic loads induced by humans: A literature review
}

\author{
Vitomir Racic*, Aleksandar Pavic and James Mark William Brownjohn \\ Department of Civil and Structural Engineering, University of Sheffield, Sheffield, UK
}

Received 24 January 2011

Revised 17 February 2012

\begin{abstract}
This paper provides a critical overview of available technology and facilities for determining human-induced dynamic forces of civil engineering structures, such as due to walking, running, jumping and bouncing. In addition to traditional equipment for direct force measurements comprising force plate(s), foot pressure insoles and instrumented treadmills, the review also investigates possibility of using optical motion tracking systems (marker-based and marker-free optoelectronic technology) and non-optical motion tracking systems (inertial sensors) to reproduce contact forces between humans and structures based on body kinematics data and known body mass distribution. Although significant technological advancements have been made in the last decade, the literature survey showed that the state-of-the-art force measurements are often limited to individuals in artificial laboratory environments. Experimental identification of seriously needed group- and crowd-induced force data recorded on as-built structures, such as footbridges, grandstands and floors, still remains a challenge due to the complexity of human actions and the lack of adequate equipment.
\end{abstract}

Keywords: Ground reaction force, vibration serviceability, human induced vibrations, footbridges, grandstands, floors, staircases

\section{Introduction}

Human-induced vibrations have become an increasingly critical factor in serviceability and safety aspects of structural design in the last decade. There is a rapidly growing number of reported problems related to vibration performance of floors [1], entertainment venues [2], footbridges [3] and staircases [4] due to people walking, running, jogging, bouncing and jumping. The lack of adequate formal design guidance regarding vibration assessment of civil engineering structures when occupied and dynamically excited by people is a consequence of lack of attention paid to this form of loading and lack of fundamental data for many relevant load case scenarios, particularly for group and crowd situations. In fact, when compared with other key dynamic loads of civil engineering structures, such as due to waves, wind and earthquakes, the current knowledge about human-induced dynamic loading is still rudimentary.

This cross-disciplinary literature survey aims to provide a critical review of the available technology and facilities for experimental characterisation of human-induced dynamic forces and focus on their application in the structural dynamics context. Section 2 covers test facilities for direct force measurements, i.e. force plates, insole pressure sensors and instrumented treadmills, which are commonly used in biomechanical studies of human gait. Transferred and adapted from animation and film industry, robotics and aerospace engineering, motion tracking technology and its utilisation to measure indirectly human-induced loads on structures is discussed in Section 3. Finally, the key findings from this literature survey are summarised in Section 4.

*Corresponding author: Vitomir Racic, Lecturer in Structural Engineering, Department of Civil and Structural Engineering, University of Sheffield, Sir Frederick Mappin Building, Sheffield S1 3JD, UK. Tel.: +44 (0) 114222 5727; Fax: +44 (0) 114222 5700; E-mail: v.racic@ sheffield.ac.uk. 


\section{Direct force measurement}

Human-induced excitation of structures derives from the contact force between the feet and the supporting structure, hence it is generally known as ground reaction force (GRF). All devices for direct measurements of GRF can be divided into three groups: force plates (Section 2.3), instrumented treadmill devices (Section 2.4) and pressure insoles (Section 2.5). Before elaborating their technical details, the next two sections provide an overview of the general principles of force transducers used by all three groups.

\subsection{Force transducers}

Force transducers are sensing elements which can measure external forces applied to deformable materials [5]. Two types of force transducers are widely used nowadays: resistive and piezoelectric. The former type can serve as resistors within a circuit such as a Wheatstone bridge where deformation under a load applied causes structural and geometric changes in resistors resulting in changes in their electrical resistance [5]. These translate directly to changes in voltage which are proportional to force changes.

A different type of sensing element is the piezoelectric crystal, naturally occurring mineral quartz, which when subjected to mechanical load generates an electric charge in response to deformation from the load applied. This physical relationship is generally known as the piezoelectric effect [6]. Although the associated electronic circuit is very different from that in the resistive case, the concept is the same. Piezoelectric crystals produce a measurable electric charge proportional to the mechanical load applied: the higher the load, the higher the charge [6].

\subsection{Properties of force transducers}

Force transducers can be selected based on their inherent properties, such as linearity, sensitivity, range, frequency response and natural frequency [5]. Both resistive and piezoelectric force transducers cannot satisfy equally well all these properties. Piezoelectric sensors have the more stable mechanical and electrical properties over a wide temperature range and virtually no deterioration with age. Wide measuring range and sensitivity, high natural frequency, good linearity and high electrical insulation resistance are their further advantageous characteristics [6]. However, the output force signals always exhibit a slight drift when measured over several hours or days and cannot be avoided. Drift is defined as an undesirable change in output signal over an extended period of time that is not a function of the measured input [6]. Resistive sensors are less expensive and have good static capabilities and negligible drift, but do not have the long-term stability and the range and sensitivity of piezoelectric models. Decision to use resistive or piezoelectric transducers in a study of human-induced forces depends primarily on the cost and human activity being measured, as explained in the next section.

Modern devices for direct measurements of human-induced forces commonly utilise multi-axial transducers which can measure forces and often also moments in three orthogonal directions [6-8]. The material of the measuring device in which transducers are installed may have considerable elasticity that transmits effects of a force acting along one orthogonal axis in the direction of other two orthogonal axes, which should not happen. This effect is generally known as crosstalk [5]. One way to compensate for this undesirable effect is to use a calibration matrix which relates the voltage response of the transducer in all three directions to given input force applied purely in one direction [7].

\subsection{Force plate}

Force plates, also known as force platforms, are devices designed to measure GRFs generated by people standing and moving on them or moving across them. General principles behind force plates depend mainly on the type of force transducers (i.e. resistive or piezoelectric) built in the platform to measure GRFs (Fig. 1).

In three-dimensional (3D) space the resultant force and moment vectors can be resolved into three components acting along three-axial orthogonal coordinate system (Fig. 2). Conventionally, the axes of the coordinate system are vertical $\mathrm{Z}$, along the length of the plate $\mathrm{Y}$, and across the width of the plate $\mathrm{X}$. The associated force and moment components are thus called $\mathrm{F}_{x}, \mathrm{~F}_{y}, \mathrm{~F}_{z}$ and $\mathrm{M}_{x}, \mathrm{M}_{y}$ and $\mathrm{M}_{z}$. When designing structures which are 


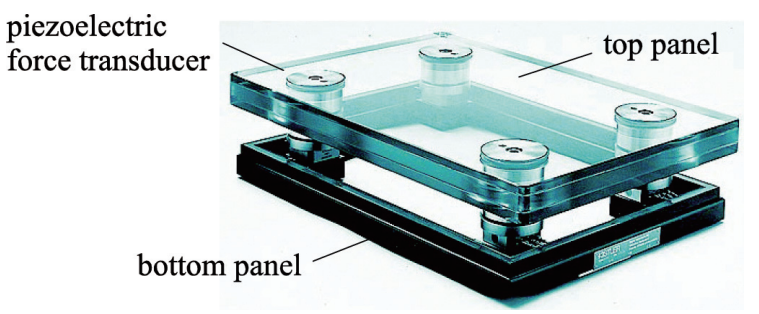

Fig. 1. Example of a Kistler force plate design [6].

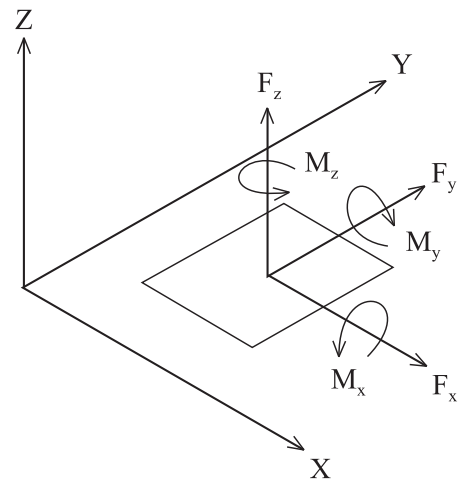

Fig. 2. Three-axial orthogonal force plate coordinate system and associated force and moment components (after Caldwell et al. [5]).

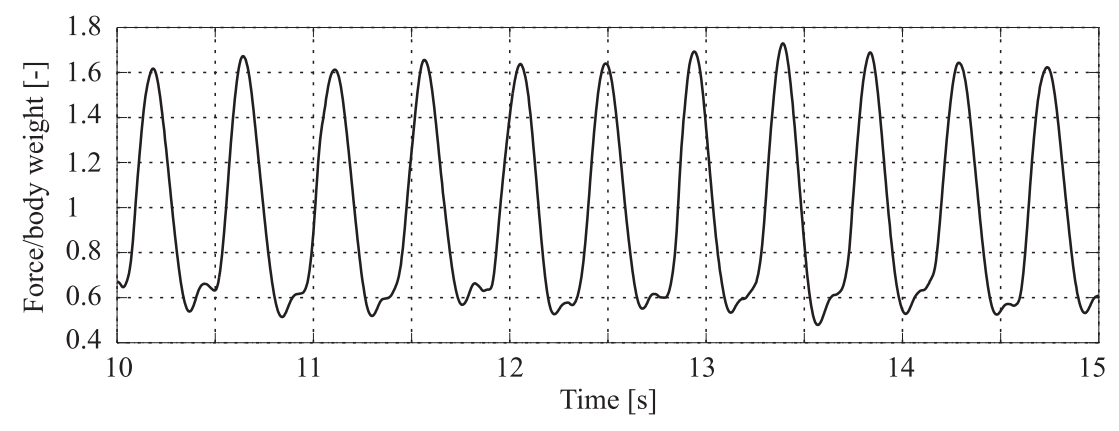

Fig. 3. Vertical force-time history due to a single person bouncing.

predominantly occupied by active humans, civil structural engineers typically need only vertical $\mathrm{F}_{z}$ and/or lateral $\mathrm{F}_{x}$ force components (e.g. in the case of footbridges).

Selecting a force plate depends primarily on two aspects: human activity under consideration (bouncing, jumping, walking and running) and target test subjects (children and adults). The selection should start with making a decision about size of a plate which will suit best these two aspects. This is followed by selecting range and natural frequency, as is outlined in the next three sections.

\subsubsection{Dimensions}

Standard dimensions (typically $600 \times 400 \mathrm{~mm}$ and $600 \times 600 \mathrm{~mm}$ ) are well sized to accommodate a wide range of children and adults bouncing, i.e. moving the upper part of the body up and down whilst the feet are in the permanent contact with a plate's top surface. A portion of a typical vertical force time history generated by a single test subject bouncing is given in Fig. 3 .

However, the size of force plates often place restrictions on studies of jumping forces. This is because when jumping, people move in all directions to keep balance more efficiently, so they might miss the plate while jumping. On the other hand, paying too much attention to targeting a limited jumping area can influence the natural jumping manner thus alter the natural variability of the corresponding GRFs [9-11]. Therefore, plates with bigger measuring surface are preferable in the studies of people jumping. A portion of a typical vertical force measurements generated by a single test subject jumping is illustrated in Fig. 4.

In the case of walking and running, a single force plate can measure a single footfall only [12], as shown in Fig. 5. However, natural variability is present in all human movement, thus a single footfall may not represent the individual's long-term performance $[9,13]$. Several successive steps reflect the real walking/running better. A simple solution to this problem is to stagger multiple force platforms in a walkway arrangement (Fig. 6) which can be spaced to accommodate several successive steps of a wide range of test subjects. As the step length is directly proportional to walking/running speed $[9,10]$, maximum speed and target number of footfalls define the length of a walkway. 


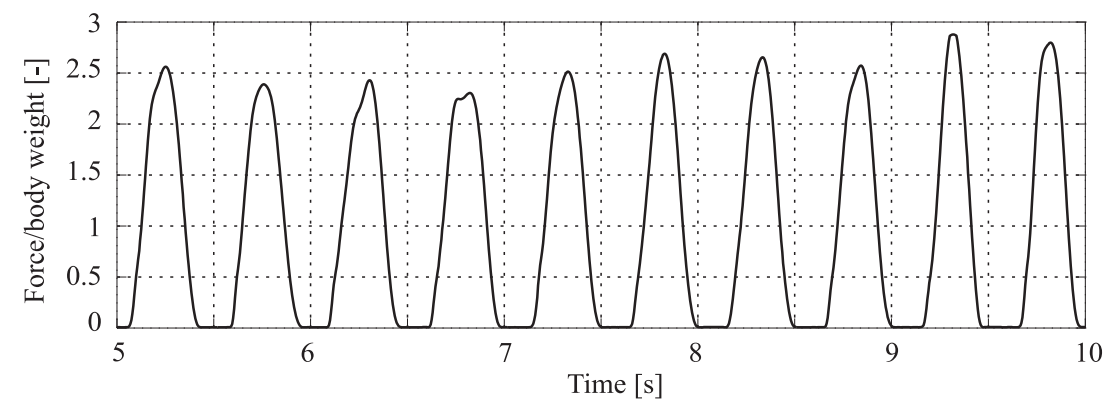

Fig. 4. Vertical force-time history due to a single person jumping.

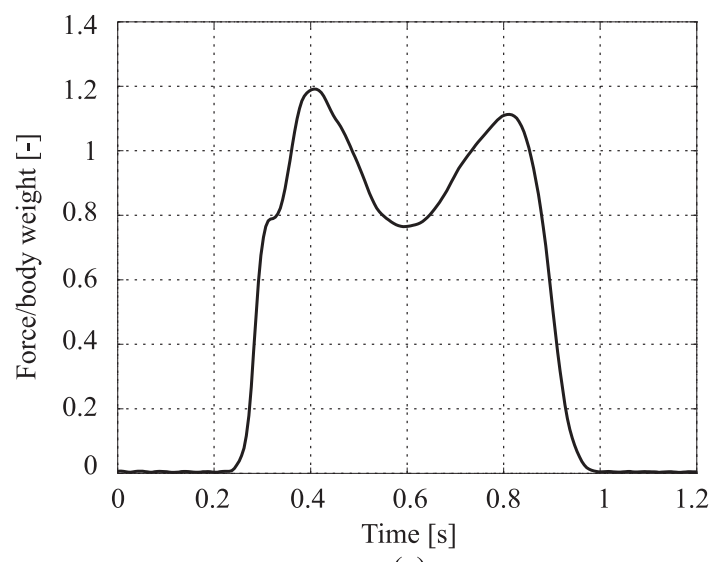

(a)

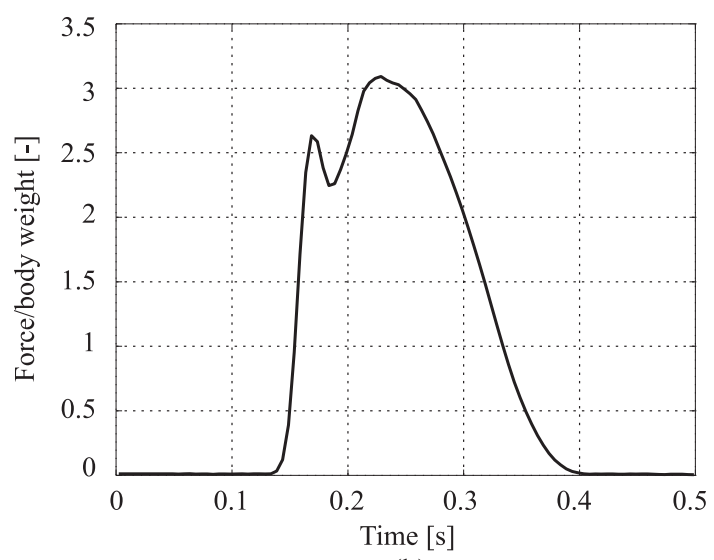

(b)

Fig. 5. Vertical GRF measured by a force plate due to a single person (a) walking and (b) running.

For instance, the arrangement illustrated in Fig. 6 enables a minimum of three footfalls to be captured at the fastest walking speeds. However, the problem with controlling and targeting footsteps to land at particular locations of the force plates remains unsolved. This multiunit arrangement also requires considerable laboratory space. Alternative devices, such as instrumented treadmill and insole pressure sensors, designed to overcome problems associated with targeting, limited number of successive steps and lack of laboratory space are presented in Sections 2.4 and 2.5.

The load capacity (also called range) is the highest load that a force platform can measure. Most strain gauge platforms are available in 1000, 2000 and $4000 \mathrm{lb}$ capacities (approximately 4.5, 9 and $18 \mathrm{kN}$ ). Research on walking forces has shown that the maximum $\mathrm{F}_{z}$ loads can be in the order of $140 \%$ of body weight [12]. Therefore, if the platform is being used strictly in the studies of humans walking, the $1000 \mathrm{lb}$ capacity platforms will provide the best resolution. However, take-off and landing phases of jumping and running produce large impulsive forces which are potentially three to four times the body weight $[15,16]$ leading to the choice of high capacity plates.

In contrast, piezoelectric force plates with quartz crystal sensors (e.g. Kistler) can accurately measure both very small and very large forces within their structural load capacity. It is therefore not necessary to purchase different piezoelectric force plates to cover several measuring ranges to provide the best resolution for all activities. However, charge amplifiers of piezoelectric plates always exhibit a slight drift for measurements carried out over several hours, as explained in Section 2.2.

\subsubsection{Natural frequency}

A platform fundamental natural frequency of between five to ten times the highest frequency of interest will adequately protect the measurements from the error associated with resonance [7]. The high rigidity of quartz crystal sensors is reflected in the very high overall stiffness of piezoelectric force plates, which results in a very high natural frequency and damping in all three directions of measurement [6]. However, they are considerably more 
Table 1

Comparison between strain gauge and piezoelectric force platforms

\begin{tabular}{lll}
\hline Property & Strain gauge & Piezoelectric \\
\hline Capacity & Limited range & Wide range \\
Frequency response & Low frequency response & High frequency response \\
Accuracy & Accurate & More accurate \\
Drift & No drift & Always present \\
Calibration & Needs regular balancing & No balancing \\
Cost & Inexpensive & Expensive \\
\hline
\end{tabular}

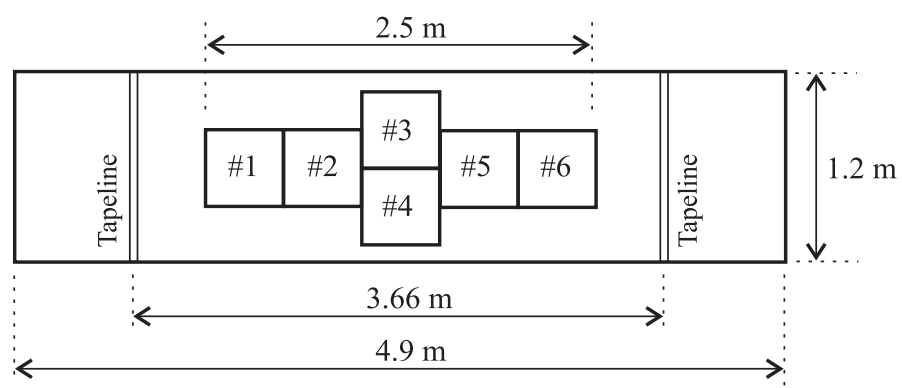

Fig. 6. A combination of six force plates (numbered 1-6) arranged along the walkway to measure several successive footfalls (after Gard et al. [14]).

expensive relative to force plates with strain gauge sensors. A summary of the comparison between strain gauge and piezoelectric force plates is given in Table 1.

\subsubsection{Capacity}

Force platforms behave essentially like accelerometers and produce outputs from the floor vibrations [7] due to inertia forces coming from the motion of the underlying floor, which can be significant if the supplying surface is flexible (range of few mm not $\mu \mathrm{m}$ ). This means that the force measured is the sum of the GRF and the inertia force calculated as effective mass of the force plate connected to the force transducers (typically the top surface of the plate) and its acceleration. Hence, heavy concrete construction or concrete slabs on grade provide the best flooring situations for their mounting.

\subsection{Instrumented treadmill}

As interest in time-varying walking and running forces broadened over the last ten years, new tools have been developed for continuous and equally accurate measuring of these forces during a large number of successive cycles and over a wide range of steady-state gait speeds [17]. These tools are based on a treadmill equipped with force sensing elements, hence generally known as instrumented force measuring treadmills (IFMTs).

\subsubsection{Single belt design}

The majority of commercially available treadmills have a single belt which provides a common walking/running area for the left and right feet (Fig. 7). Various designs have been proposed, including mounting a force platform inside the treadmill [18] and building the treadmill around a floor-mounted force plate [19]. As such, they can measure the sum of GRFs acting under the both feet. However, a problem typically appears when the left and right footfalls are to be studied separately. In this case, the above mentioned designs are suitable for assessment of running forces only. This is because left and right running GRFs are clearly separated with zero force periods corresponding to aerial (often called 'flying') phase of running (Fig. 8(a)). However, it is difficult to distinguish between left and right forces during the double support phases of walking, i.e. a period in a walking cycle when both feet are in contact with the ground (the shaded area in Fig. 8(b)). Two solutions were initially presented. Davis and Cavanagh [20] offered an algorithm that extracts left and right GRF traces from the common signal. The alternative was a new treadmill design including two force plates under the belt [21]. One force plate was placed in front and one at the 


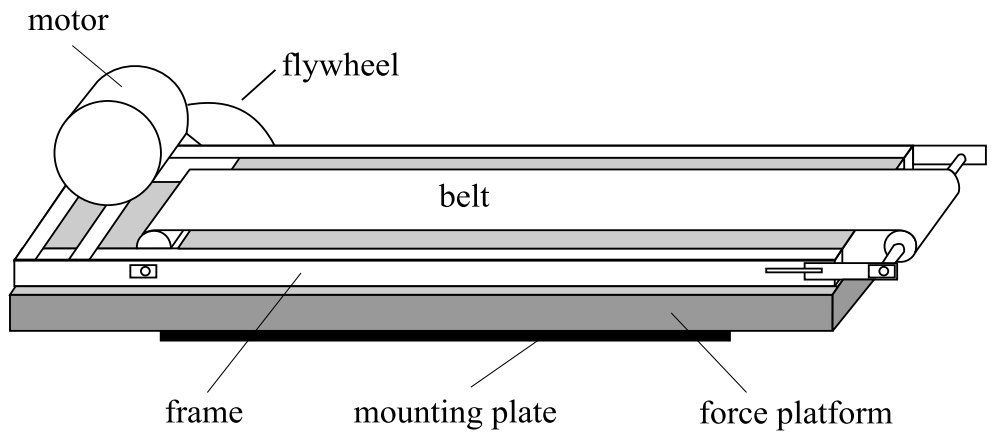

Fig. 7. Example of single-belt treadmill design (after Kram et al. [18]).

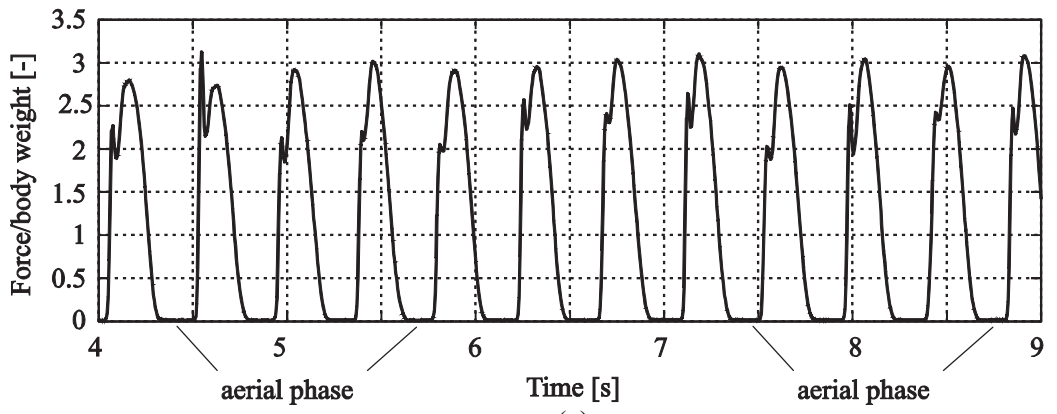

(a)

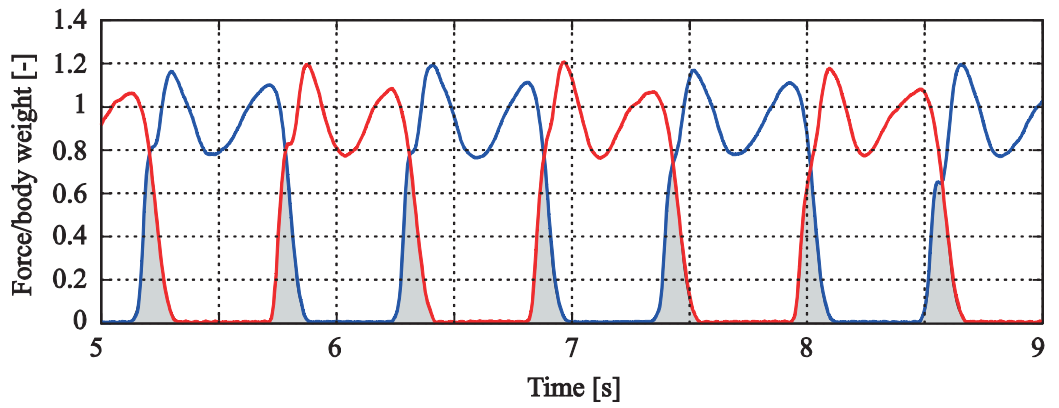

(b)

Fig. 8. Time-varying vertical GRF due to (a) running and (b) walking; the red and blue signals correspond to the left- and right-foot GRF, whereas the shaded areas represent double support phases.

back of the treadmill, assuming that only one foot would be on each force plate during the double support phase. However, this is not true for a wide range of test subjects, particularly when measuring forces generated by children and elders [22]. Moreover, designs featuring force plate(s) built under a moving belt can measure accurately only the vertical force components. This is because the horizontal force data are modified by friction forces between the moving belt and the fixed force platform(s). Nevertheless, such treadmills are usually equipped with budget uni-axial force transducers, fixed to measure vertical pedestrian forces, making them significantly less expensive than their tri-axial counterparts.

\subsubsection{Double belt design}

To measure independently the left and right footfalls, double belt design splits the walking surface into two identical IFMTs - left and right, placed close together (Fig. 9). Each treadmill belt is usually driven by a brushless servomotor equipped with internal velocity controllers (e.g. optical transducers) to maintain the speed as constant as possible. 


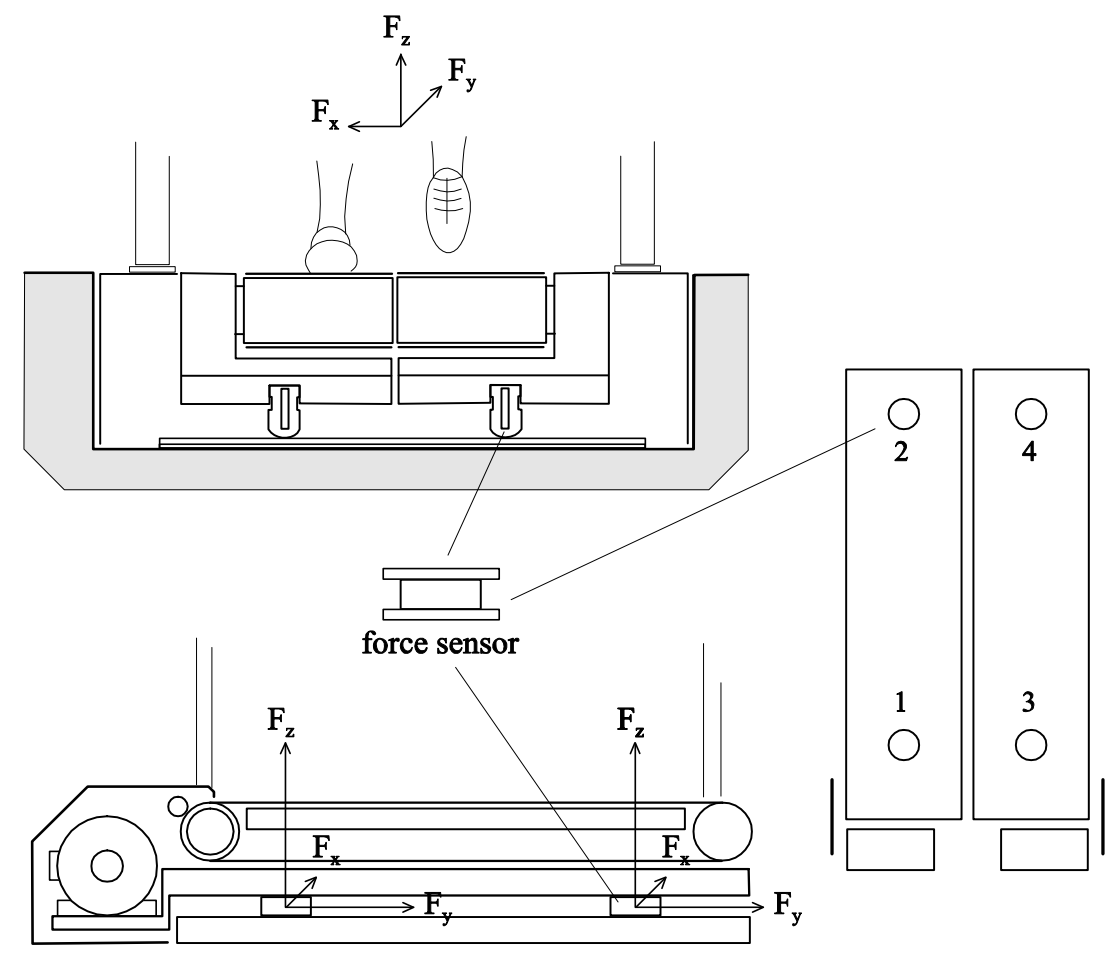

Fig. 9. ADAL3D-F concept.

In the case of so called Belli's design [17], such as ADAL3D-F [23], all components (including the motor, belt and frame) of each treadmill are mounted on a single metal frame and mechanically connected to the supporting ground only through a pair of three-axial piezoelectric force sensors. The main concern is keeping the natural frequency of the treadmill frames as high as possible [17] so that it approximates a rigid body supported by the force cells. This is achieved by a stiff steel frame which maintains a 'rigid' connection between treadmill components. Hence, assuming the rigidity of the above mentioned ensemble, the entire treadmill is mechanically isolated. This means that the forces due to belt friction and belt rotation can be considered as internal forces and are not detected by the sensors. The sensors measure only external forces, i.e. actual three-axial forces exerted by the feet on the treadmill belts (Fig. 10). In the case of a flexible base structure, the external force becomes the sum of the GRF and inertia of the effective treadmill mass, as already explained in Section 2.3.3. Therefore, for the highest accuracy measurements the treadmill should be mounted in a manner that minimizes vibration of the entire treadmill system. Similarly to force plates, this is best accomplished by fixing the treadmill to a solid concrete floor structure. Mounting in a recessed pit illustrated in Fig. 9 is a common solution for ground floor installations.

Because of their limited dimensions, both force plates and instrumented treadmills can measure only individual force data. However, a single occupant on a large structure is far from the expected loading scenario in the majority of cases. Examples are entertaining venues during concert events, shopping malls and footbridges in busy city centres, which are predominantly occupied and dynamically excited by multiple persons. The remaining part of this paper presents technology which can simultaneously measure forces induced by each group member. Technology for making such measurements is currently very limited yet of great importance for developing mathematical models of group and crowd loading. This is because they contain essential information to study synchronisation effect between different group members $[13,16]$.

\subsection{Foot pressure insoles}

Other direct methods used by biomechanics researchers to measure GRFs include pressure sensors attached to insoles, hence they are often called instrumented shoes [24,25]. A typical shape of the vertical pressure distribution 


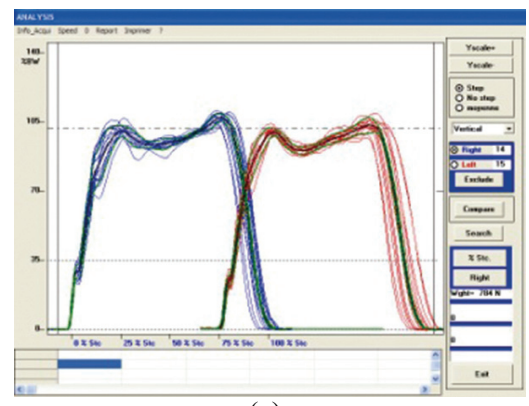

(a)

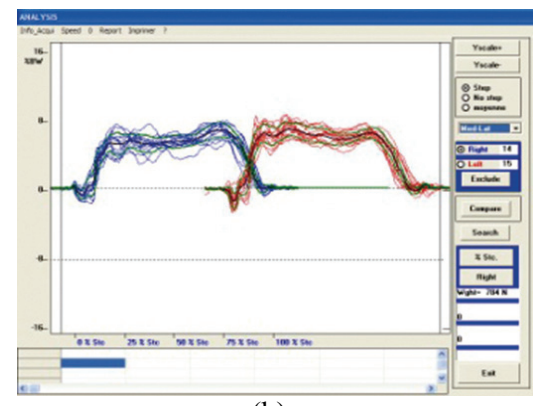

(b)

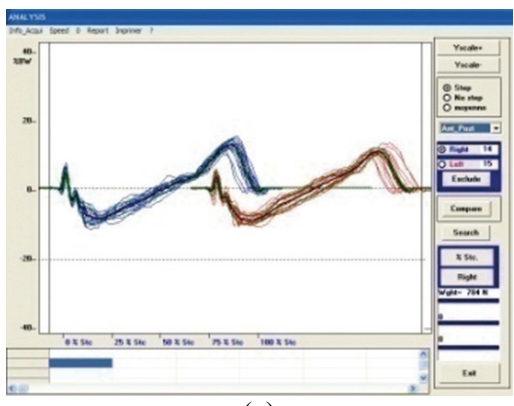

(c)

Fig. 10. Force records in (a) vertical, (b) lateral and (c) longitudinal direction for a single test subject measured by the ADAL3D-F during one walking exercise. Left (red) and right (blue) individual steps are overlapped using Adisoft2000 [22].

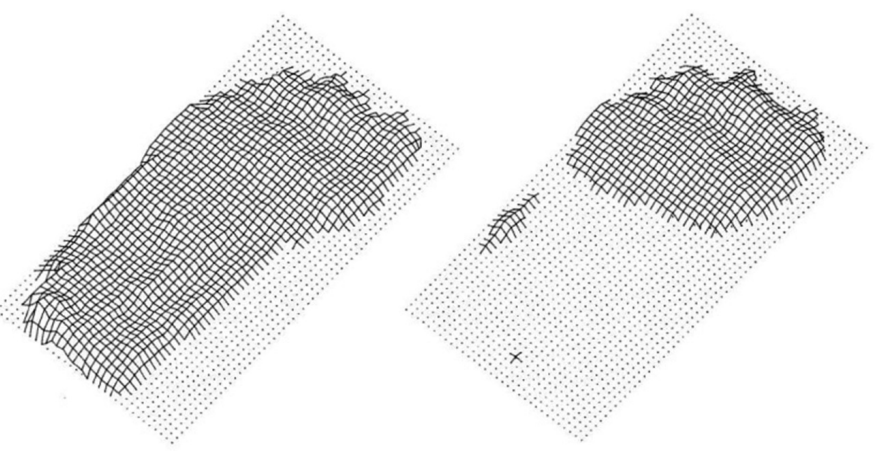

Fig. 11. Pressure patterns from the left foot at different times during the contact phase of running (after Caldwell et al. [5]).

for a subject running is given in Fig. 11. The GRF amplitude can be calculated as area integral of the distributed pressure. Typically, only vertical GRF is reported [26], although several researchers proposed algorithms to determine the horizontal forces having measured the vertical pressure distribution [24,25]. Apart from direct measurements of a group forces, the key advantage of pressure insoles is the ability to record GRFs for consecutive steps without imposing constraints on foot placement, such as targeting the footsteps in the case of a force plate or constrained walking speed in the case of a treadmill. However, when compared with a force plate and treadmill data, pressure insoles data universally lack accuracy of the force amplitudes [27]. In the civil engineering context, the unique attempt involving insole pressure sensors during the Millennium Bridge investigation [3] was never publicised in more detail, so it is not clear how successful it was.

\section{Indirect force measurement}

Motivated by several biomechanical studies designed to estimate contributions of motion of various body segments to GRFs [28-30], the authors recently developed a novel method in civil engineering context to indirectly measure vertical dynamic loading induced by small groups jumping and bouncing on full scale structures [16]. The method rests on the basic principles of Newtonian mechanics. Broadly speaking, the dynamic force a person generates against the ground must react against inertia of their body, so that the sum of products of masses and accelerations for all body parts must equal the force at all times:

$$
\boldsymbol{F}=\sum_{i=1}^{s} m_{i}\left(\boldsymbol{a}_{i}-\boldsymbol{g}\right)
$$

Here, $\boldsymbol{F}=\left\{F_{x}, F_{y}, F_{z}\right\}$ is dynamic force vector, $m_{i}$ and $\boldsymbol{a}_{i}=\left\{a_{i x}, a_{i y}, a_{i z}\right\}$ are mass and acceleration vector of the centre of the mass of the i-th body segment (assumed to be rigid) and $\boldsymbol{g}=\{0,0,9.81\} \mathrm{m} / \mathrm{s}^{2}$ is the vector of static acceleration due to gravity. 


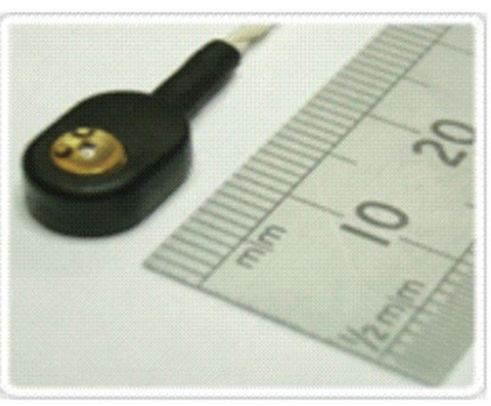

(a)

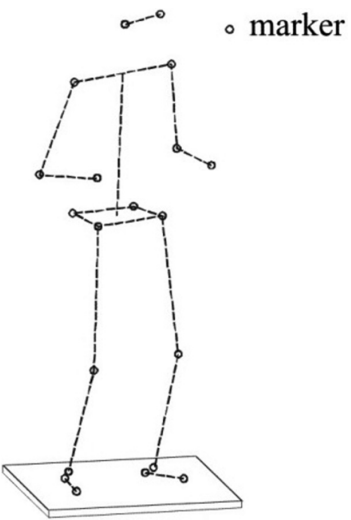

(b)

Fig. 12. (a) Motion tracking marker. (b) Body model and marker arrangement.

To estimate mass and its centroid for each segment, several biomechanical methods have been proposed so far. Regression equations generated from cadaver data [31,32] and data from live humans [33,34] have been the most popular analytical approach, mainly because they can provide estimates of these parameters quickly and easily. A more detailed summary of available anthropometric models can be found in a literature review article by Racic et al. [13].

Vertical accelerations of the body segments in Racic et al. [16] were measured using tracking optical markers (also called "target markers") stuck to the skin's surface (Fig. 12). The markers were placed in a manner to satisfy technical requirements, such as high visibility from sensors and to minimise relative displacement between them and underlying bone during movement, generally known as "soft tissue artefact" [36]. The latter is important since Eq. (1) works under the assumption of rigid body segments [16].

Results showed that the method could reproduce reliably the loading amplitude spectra below approximately $15 \mathrm{~Hz}$, thus can be used successfully in studies of the negative cue effect of a perceptibly vibrating structures on GRFs, energy flow and power in the human-structure system, and also synchronisation between individuals in a group. However, reliable reproduction of the higher frequency content is hardly possible due to the soft tissue artefact. The effect is dominant during abrupt contacts between the feet and supporting structure, such as the landing phase of jumping, yielding noisy motion data.

Bouncing and jumping force harmonics at frequencies higher than $15 \mathrm{~Hz}$ are rarely relevant in structural design. However, energy of walking excitation at frequencies as high as $50 \mathrm{~Hz}$ can cause excessive micro vibrations of floors accommodating vibration sensitive equipment requiring sub-micron positional precision [13], such as facilities for particle physics research and lasers in eye clinics. In addition, ability to reproduce low frequency range of lateral and longitudinal walking loads still remains unproven. Most motion capturing systems use optoelectronic technology to quantify body kinematics, as explained in Sections 3.1-3.3. Alternative non-optical systems, such as wireless inertial sensors, are presented in Section 3.4.

\subsection{General principles of marker-based optoelectronic systems}

Motion tracking markers can be either passive (reflective) or active (light emitting). The core of passive marker technology is a set of cameras equipped with infrared light emitting diodes (LED) mounted around the camera lens. These illuminate the markers with stroboscopic rays of light which reflections are recorded as video frames [37]. The video frames (also known as video images) need additional processing using pattern recognition software [38] or specialised hardware [39] to identify the positions of each marker. This is because each of the cameras can provide only a set of two-dimensional (2D) passive marker coordinates placed in the plane perpendicular to its optical axes [40]. If at least two cameras see the same marker, the 3D coordinates can be obtained using the geometrical properties of central projection and mathematical triangulation [40]. This is similar to the human brain which uses input from both eyes to perceive depth in the field of vision. Multiple units may be combined to maximise visibility 
of passive markers and to give even greater capture volume, i.e. the volume of space within which markers are visible to cameras thus often called the '3D volume'. Typically, a passive tracking system will consist between six to twenty four cameras. The number of cameras depends primarily on the total number of tracking markers, number of tracking subjects and target objects (e.g. full body versus only few body segments). In film industry, there are examples of using over three hundred passive cameras to capture accurately full body kinematics of only two persons [37].

On the other hand, active optoelectronic systems utilise self-illuminated markers. Each active marker is an LED that pulses sequentially and is activated at time instances differenting by a few microseconds [13]. This time shift enables optic sensors to detect positions of markers independently, i.e. each marker is given a unique identity, so no software processing is required to identify and determine individual marker positions. Moreover, a single sensor is enough to measure the locations of active markers in the 3D with high resolution and accuracy (errors in a marker position are typically less than $1 \mathrm{~mm}$ ). This is because each sensor has multiple cameras. For example, each Codamotion sensor [41] and Optotrak Certus sensor [42] contain three cameras. As in the case of passive motion capture systems, extra sensors are required for full body kinematics of multiple subjects and a bigger 3D volume.

\subsection{Comparison between passive and active marker technology}

Although passive marker systems, such as Vicon [37] and Qualisis [43], have the advantage of using lightweight reflective markers without wires and batteries on the body of the test subject, their accuracy usually cannot reach the standards of their active marker counterparts [44]. Even if markers are temporarily lost from a field of view, active marker systems can maintain the identification of markers automatically by interpolation and optimisation algorithms. Also, contrary to passive systems, virtual merging of markers when their trajectories cross cannot occur with an active system, so the markers can be placed close together which is their very important advantage.

The authors used successfully active Codamotion system to track body motion of people in a laboratory environment [16]. However, to the best knowledge of the authors, there is no study which used marker-based systems for monitoring people in outdoor environments, which is very important application in civil engineering dynamics. Therefore, based on the past experience and discussions with other researchers from many cross-disciplinary areas, such as biomechanics, biomedicine, animation and robotics, the authors can only point out several major obstacles of optoelectronic technology when monitoring people in natural scenes:

1) Tracking full body kinematics of more than two individuals is problematic, with close interactions between group members resulting in significant marker occlusions. Additional tracking sensors can improve significantly the visibility of markers but this becomes too costly as group size increases.

2) Optoelectronic systems interact with sunlight, which causes problems when tracking markers in outdoor environments. A possible solution to the problem is to carry out experiments during night, which raises a question of biased data sample.

3) Both reflected light from passive markers and infrared signals from active markers become very noisy when markers are far away from cameras/sensors. Since cameras/sensors must be at fixed and stable positions to ensure that they do not move relative to the markers, monitoring people on long-span structures, typically footbridges for which the only fixed points are at the nearby ground, can be too difficult.

4) Marker placement can be time-consuming, so that participants tire, become impatient and lose interest in experiments.

Eliminating the need for markers would enable simple, time-efficient and potentially more meaningful assessments of human movement. In the civil engineering context, this particularly addresses monitoring people in their natural environments, such as offices, shopping malls and bridges. Utilisation of computer vision and wireless inertial sensors for such purposes seems to be the way forward and is presented in the next two sections.

\subsection{Markerless optoelectronic systems}

Video camera tracking without the aid of markers is considered by many researchers as the ultimate solution to human motion tracking challenges $[45,46]$. Although computer-vision-based motion tracking has become an active 
research area in the last decade, markerless methods are not widely available because accurate capture of human movement without markers is technically challenging.

The state-of-the-art of existing methods is based on three major tasks: human detection, motion tracking and behaviour understanding. Human detection involves segmenting regions corresponding to people from the rest of a video frame [45]. It is of fundamental importance for human motion analysis since the subsequent tasks greatly depend on it. However, changes in weather, lightning and moving objects, such as vehicles, flying birds and flowing clouds in natural scenes, make detection of target objects (here humans) difficult [47]. For example, the shadow distorts the body shape making the shape recognition less reliable. Four methods for detection are used: background subtraction, statistical methods, temporal differencing and optical flow. The reader is referred to a comprehensive survey article by Wang et al. [45] for a full explanation of these methods.

As already mentioned, the best method of human motion tracking for application in the civil engineering context would ideally enable detection and tracking of multiple people under congested conditions in natural environments. However, the majority of present computer vision systems, such as IMAGO [48], cannot effectively handle the problems of self-occlusion (i.e. differentiation between different body parts) and mutual occlusions between people and/or objects. For example, people shaking hands and dancing may be classified as a single person/object. Typically, during occlusions, only portions of each person are visible and often at very low resolution [49]. A notable progress has been made using statistical methods [50], which essentially attempt to predict body pose, position of body segments, etc. from available video frames. Perhaps more effective practical method for addressing occlusion is increasing the number of tracking video cameras [45]. Multiple viewpoints also allow 3D tracking, which can provide better insight into human actions. However, vision-based 3D tracking is still at its infancy due to high computational complexity [46].

Motion tracking over time typically involves matching body segments in successive video frames using features such as points, lines or blobs, and their position, velocity, shape, texture, colour, etc. Tracking can be classified into various categories according to different criteria. Regarding target objects, tracking can be focused on specific body parts, such as head, trunk and limbs [51], and tracking of the whole body [52]. If the number of cameras is considered, there is single camera tracking [53] and multiple camera tracking [54] of moving people. Further classification can be made according to the dimension of tracking space (2D and 3D), number of tracked people (single humans, groups and crowds) and tracking environment (indoors and outdoors). Different tracking methods are extensively summarised in survey articles by Wang et al. [45], Moeslund and Granum [49] and Moeslund et al. [55].

Behaviour understanding aims to recognise, extract and describe human motion patterns from video frames. It may be simply considered as a classification problem, i.e. matching a set of experimentally measured data with a group of template feature data representing typical human actions. For example, W4 system [56] can recognise some simple actions, such as carrying and depositing an object. This task brings a number of challenges such as how to make the templates from available samples and how to make the templates more robust, thus enabling them to cope effectively with subtle spatial and time variations within similar classes of motion patterns. The general analytical methods used in this research are dynamic time warping [57], hidden Markov models [58], neural networks [59] and state-space approaches [60]. Still at a rudimentary stage, understanding human behaviour is the most interesting long-term research niche in video-based human motion analysis.

\subsection{Non-optical motion tracking systems}

Recent advances in microelectronics and wireless technology have made it possible to build cordless inertial sensors that are both small and light enough to be unobtrusive for a number of sporting, clinical, military, aerospace, automative industry and civil engineering applications [61]. Micro-Electro-Mechanical Systems (MEMS) inertial sensors (Fig. 13) consist of miniature devices that combine electrical and mechanical inertial sensing components [62]. They contain miniature masses and sensing electronics which respond to minute changes in inertia in the linear directions (acting as accelerometers) and radial directions (acting as gyroscopes).

These accelerometers typically use a suspended silicon mass on the end of a silicon arm (also called 'fork') that has a single degree of freedom [63]. Motion of the suspended mass causes deformation of the silicon arm, 


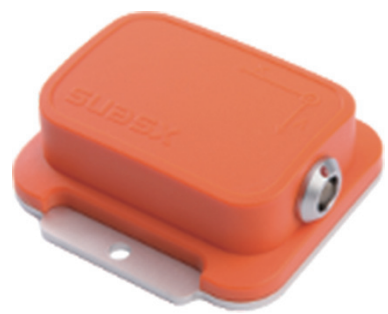

(a)

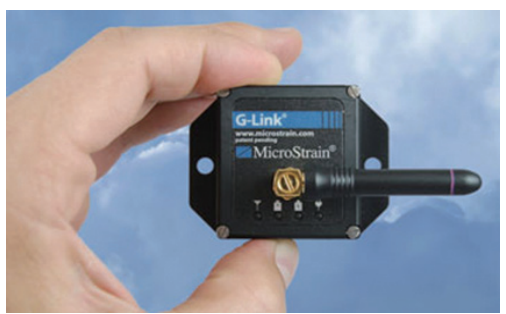

(b)

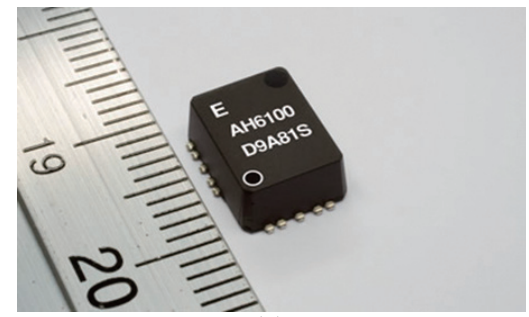

(c)

Fig. 13. Examples of MEMs (a) Xsens, (b) MiocroStrain, Inc (c) Epson Toyocom Corporation.

which is detected by piezoelectric elements embedded in the arm and transformed to acceleration output. Multiple accelerometers are typically packaged together orthogonally to offer multi-axial measurements.

Accelerometers measure the linear acceleration in the inertial reference frame, i.e. in directions that can only be measured relative to the body segment they are attached to, but are not aware of their own orientation. However, they can be used to determine orientation with respect to the earth's gravity as components of gravity are measured as DC levels [64]. This information can be used to calculate vertical accelerations of the segment in the global coordinate frame, which is exactly what marker based systems measure to reproduce vertical GRF data according to Eq. (1). Moreover, MEMS inertial sensors can offer comparable results at significantly lower prices [65], thereby decreasing the instrumentation cost of experiments. In the case when orientation of the inertial reference frame is also needed (e.g. to measure lateral body movement in the global frame to reproduce the corresponding lateral forces), rate gyroscopes can be incorporated in the measuring units [66]. For example, Xsens [67], Colibri [68] and Orient [69] successfully integrated MEMS accelerometers, gyroscopes and magnetometers to provide 3D orientation, acceleration, rate of turn and magnetic field.

Apart from being less expensive, other advantages of wireless accelerometers over optoelectronic motion capturing are:

1) there is no occlusion of sensors or line-of-sight restrictions,

2) they can be used under any lighting conditions,

3) they are portable and can be worn under normal clothing,

4) can be used in all environments (e.g. in the office, footbridge, grandstand).

However, after discussions and correspondence with other researchers with years of practical experience in using inertial sensors for detection of human motion, the authors have got an overall impression that commercially available wireless inertial measurement units commonly struggle with monitoring several individuals simultaneously. The majority of wireless units transmits data via bluetooth TM in real time which limits the number of sensors used at the same time. The common problem is data synchronisation and autonomous operation [61]. However, in structural health monitoring applications [70] there has been the emergence of so called 'smart' wireless sensors (also known as Wireless Smart Sensor Nodes - WSSNs), referring to units which have on-board memory storage and often computational capability. The measured data can be stored and processed on-board WSSN, and then transmitted to a data acquisition PC at the end of an experimental session via the wireless connection. Data synchronisation between individual sensors is achieved by synchronising their clocks at the beginning of the session. Apart from alleviating the communications burden on the network, this approach also has advantage of consuming less power [61].

\section{Summary and conclusions}

The state-of-the-art facilities for experimental identification of human-induced dynamic loads include equipment for direct measurement of the contact forces between individuals and a rigid laboratory floor, comprising a force plate and an instrumented treadmill. However, the artificial laboratory environment and constraints imposed by the direct measurement system, such as targeting footsteps on a force plate, can affect the ability of inexperienced test subjects to move naturally and therefore may alter the contact forces. Also, when dealing with issues like vibration 
serviceability of real full-scale structures, there is a need to estimate loads applied directly by multiple occupants under a wider range of conditions in their natural environments, such as an office or footbridge. Although foot pressure insoles seems to be a promising alternative to force plates and treadmills, at present many issues remain open such as accuracy of the measurements and data synchronisation between many individuals.

In addition to traditional direct force measurements, this paper also presents methods for indirect measurement of time-varying records of human-induced forces via combination of visual motion tracking (imaging) data and known body mass distribution. Compared to the direct measurements, this approach uses 'free field' measurement of human-induced forces without artificial restrictions imposed on human movement in laboratory environments. Motion tracking technology is transferred and adapted from scientific branches with the common interest in human locomotion, such as biomedicine, sports science and video animation, whereas anthropometry of the body segments is well documented in a number of medical publications.

Optoeletronic marker-based tracking systems are often used as a 'golden standard' in human motion analysis due to their accuracy. This accuracy particularly motivates their applications in medicine, biomechanics, video game and film industry, robotics and aerospace engineering. However, poor performance in outdoor environments limits their application in the civil engineering context. Alternative marker-free computer vision technologies can be used in all environments, but they face other technical challenges. Typical problems include a trade-off between computational cost and accuracy, self-occlusion of human body, occlusion between people and objects and mutual occlusions between multiple people under congested conditions. Many challenges also remain open due to the complexity of human motion and the existence of error or noise in measurement. Occlusion can be successfully handled by inertial sensors, such as wireless accelerometers. However, the majority of commercially available inertial sensors have common problems with data synchronisation and autonomous operation.

Overall, application of body motion tracking technologies in civil structural dynamics is still an open research area deserving further attention in future. It will be important to test the robustness of rapidly emerging new systems in various environments and under various group and crowd conditions. Moreover, it might be helpful and necessary to combine various technologies to advance human motion analysis to all possible situations.

\section{Acknowledgments}

The authors would like to acknowledge the financial support provided by the UK Engineering and Physical Sciences Research Council (EPSRC) for grant reference EP/E018734/1 ('Human Walking and Running Forces: Novel Experimental Characterisation and Application in Civil Engineering Dynamics').

\section{References}

[1] D. Parker, Rock fans uncover town hall floor faults, New Civil Engineer, 20 November 2003.

[2] M. Glackin, Stadia design rethink prompted by Cardiff fiasco, Building 11 January 2000.

[3] T. Fitzpatrick, P. Dallard, S. Le Bourva, A. Low, R. Ridsdill-Smith and M. Willford, Linking London: The Millennium Bridge, Report No. L12.32. London, UK: The Royal Academy of Engineering, 2001.

[4] S.C. Kerr and N.W.M. Bishop, Human induced loading on flexible staircases, Engineering Structures 23 (2001), $37-45$.

[5] G.E. Caldwell, D.G.E. Robertson and S.N. Whittlesey, Forces and their measurement, in: Research Methods in Biomechanics, D.G.E. Robertson, G.E. Caldwell, J. Hamill, G. Kamen and S.N. Whittlesey, eds, Human Kinetics, Champaign, Ilinois, USA, 2004.

[6] Kistler, Kistler product manuals, www.kistler.com, 2011.

[7] AMTI, AMTI product manuals, Advanced Mechanical Technology, Inc, Watertown, USA, 2011.

[8] Bertec, Bertec force plate manual, Bertec Corporation, Columbus, Ohio, USA, 2011.

[9] J. Perry, Gait analysis: Normal and pathological function, Thorofare, New York, USA, 1992.

[10] D.A. Winter, Biomechanics and motor control of human movement, $2^{\text {nd }}$, John Wiley and Sons, eds, Toronto, Canada, 1990.

[11] V. Racic, A. Pavic and J.M.W. Brownjohn, Number of successive cycles necessary to achieve stability of selected ground reaction force variables during continuous jumping, Journal of Sports Science and Medicine 8 (2009), 639-647.

[12] S.C. Kerr, Human induced loading on staircases, PhD Thesis, University College London, Mechanical Engineering Department, London, UK, 1998

[13] V. Racic, A. Pavic and J.M.W. Brownjohn, Experimental identification and analytical modelling of human walking forces: Literature review, Journal of Sound and Vibration 326 (2009), 1-49. 
[14] S.A. Gard, S.C. Miff and A.D. Kuo, Comparison of kinematic and kinetic methods for computing the vertical motion of the body center of mass during walking, Human Movement Science 22(6) (2004), 597-610.

[15] V. Racic and A. Pavic, Stochastic approach to modelling near-periodic jumping force signals, Mechanical Systems and Signal Processing24 (2010), 3037-3059.

[16] V. Racic, J.M.W. Brownjohn and A. Pavic, Reproduction and application of human bouncing and jumping forces from visual marker data, Journal of Sound and Vibration 329 (2010), 3397-3416.

[17] A. Belli, P. Bui, A. Berger, A. Geyssant and J.R. Lacour, A treadmill ergometer for three-dimensional ground reaction forces measurement during walking, Journal of Biomechanics 34(1) (2001), 105-112.

[18] R. Kram and A.J. Powell, A treadmill-mounted force platform, Journal of Applied Physiology 67(4) (1989), 1692-1698.

[19] R. Kram, T.M. Griffin, J.M. Donelan and Y.H. Chang, Force treadmill for measuring vertical and horizontal ground reaction forces, Journal of Applied Physiology 85(2) (1998), 764-769.

[20] B.L. Davis and P.R. Cavanagh, Decomposition of superimposed ground reaction forces into left and right force profiles, Journal of Biomechanics 26 (1993), 593-597.

[21] J.B. Dingwell and B.L. Davis, A rehabilitation treadmill with software for providing real-time gait analysis and visual feedback, Journal of Biomechanical Engineering 118 (1996), 253-255.

[22] F. Dierick, M. Penta, D. Renaut and C. Detrembleur, A force measuring treadmill in clinical gait analysis, Gait Posture 20(3) (2004), 299-303.

[23] HEF Medical Development, User manuals, Lion: HEF Group, 2011.

[24] H.H. Savelberg and A.L. de Lange, Assessment of the horizontal, fore-aft component of the ground reaction force from insole pressure patterns by using artificial neural networks, Clinical Biomechanics 14 (1999), 585-592.

[25] A.F. Cordero, H.J.F. Koopman and F.C.T. van der Helm, Use of pressure insoles to calculate the complete ground reaction forces, Journal of Biomechanics 37 (2004), 1427-1432.

[26] S. Barnett, J.L. Cunningham and S. West, A comparison of vertical force and temporal parameters produced by an in-shoe pressure measuring system and a force platform, Clinical Biomechanics 16 (2000), 353-357.

[27] M. Lord, Spatial resolution in plantar pressure measurement, Medical Engineering and Physics 19 (1997), 140-144.

[28] A.B. Thorton-Trump and R. Daher, The prediction of reaction forces from gait data, Journal of biomechanics 8 (1975), 173-178.

[29] D.I. Miller and M.A. Nissinen, Critical examination of ground reaction force in the running forward somersault, International Journal of Sport Biomechanics 3 (1987), 189-206.

[30] M.M. Bobbert, H.C. Schamhardt and B.M. Nigg, Calculation of vertical ground reaction force estimates during running from positional data, Journal of Biomechanics 24 (1991), 1095-1105.

[31] W.T. Dempster, Space requirements of the seated operator, Geometrical, kinematic and mechanical aspects of the body with special reference to the limbs, Project No 7214. Aerospace Medical Research Laboratory, Wright-Petterson Air Force Base, Ohio, USA, 1955.

[32] C.E. Clauser, J.T. McConville and J.W. Young, Weight, volume and centre of mass of segments of the human body, Technical report 69-70, Aerospace Medical Research Laboratory, Wright-Petterson Air Force Base, Ohio, USA, 1969.

[33] V. Zatsiorsky and V. Seluyanov, The mass and inertia characteristics of the main segments of the human body, In H. Matsui and K. Kobayashi, eds, Biomechanics VIII-B, Human Kinetic, Illinois, USA, 1983, pp. 1152-1159.

[34] P. de Leva, Adjustments to Zatsiorsky-Seluyanov's segment inertia parameters, Journal of Biomechanics 29(9) (1996), 1223-1230.

[35] C. Motion, Inc Visual 3D product manuals, www.c-motion.com, 2011.

[36] A. Leardini, L. Chiari, U.D. Croce and A. Cappozzo, Human movement analysis using stereophotogrammetry, Part 3: Soft tissue artifact assessment and compensation, Gait Posture 21 (2005), 212-225.

[37] Vicon Motion Systems, Vicon user manual, The Oxford Instruments Group, Oxford, UK, 2011.

[38] K.M. Taylor, F.M. Mottier, D.W. Simmons, W. Cohen, Jr., R. Pavlak, D.P. Cornell and G.B. Hawkins, An automated motion measurement system for clinical gait analysis, Journal of Biomechanics 15(7) (1982), 505-516.

[39] G. Ferrigno, N.A. Borghese and A. Pedotti, Pattern-recognition in 3-D automatic human motion analysis, ISPRS Journal of Photogrammetry and Remote Sensing 45(4) (1990), 227-246.

[40] A. Cappozzo, A. Leardini, U.D. Croce and L. Chiari, Human movement analysis using stereophotogrammetry, Part 1: Theoretical background, Gait Posture 21 (2005), 186-196.

[41] Charnwood Dynamics Ltd, Codamotion user manuals, Charnwood Dynamics Ltd, Leicestershire, UK, 2011.

[42] Northern Digital Inc, Optotrak certus motion tracking system manuals, NDI, Radolfzell, Germany, 2011.

[43] Qualisys AB, Qualisys optical motion capture - user manual, Qualisys AB, Gothenburg, Sweden, 2011.

[44] L. Chiari, U.D. Croce, A. Leardini and A. Cappozzo, Human movement analysis using stereophotogrammetry, Part 2: Instrumental errors, Gait Posture 21 (2005), 197-211.

[45] L. Wang, W. Hu and T. Tan, Recent developments in human motion analysis, Pattern Recognition 36 (2003), $589-601$.

[46] L. Mündermann, S. Corazza and T.P. Andriacchi, The evolution of methods for the capture of human movement leading to markerless motion capture for biomechanical applications, Journal of Neuroengineering and Rehabilitation 3(1) (2006), no pages.

[47] D.M. Gavrila, The visual analysis of human movement: A survey computer vision and image understanding, 73(1) (1999), 82-98.

[48] IMAGO Machine Vision Inc, IMAGO user manuals, Hull, Canada, 2011.

[49] T.B. Moeslund and E. Granum, A survey of computer vision-based human motion capture, Computer Vision and Image Understanding 81(3) (2001), 231-268.

[50] G. Rigoll, S. Eickeler and S. Muller, Person tracking in real world scenarios using statistical methods, Proceedings of the International Conference on Automatic Face and Gesture Recognition, Grenoble, France, March 2000, pp. 28-30.

[51] D. Meyer, J. Psl and H. Niemann, Gait classification with HMM's for trajectories of body parts extracted by mixture densities, Proceedings of British Machine Vision Conference 11, Southampton, UK, (November 1998), pp. 459-468. 
[52] S. Corazza, L. Mundermann, A.M. Chaudhari, T. Demattio, C. Cobelli and T.P. Andriacchi, A markerless motion capture system to study musculoskeletal biomechanics: Visual hull and simulated annealing approach, Annals of Biomedical Engineering 34(6) (2006), 1019-1029.

[53] I.A. Karaulova, P.M. Hall and A.D. Marshall, A hierarchical model of dynamics for tracking people with a single video camera, Proceedings of British Machine Vision Conference 11-14, Bristol, UK, (September 2000), pp. 352-361.

[54] A. Utsumi, H. Mori, J. Ohya and M. Yachida, Multiple-viewbased tracking of multiple humans, Proceedings of the International Conference on Pattern Recognition 17-20, Brisbane, Australia, (August 1998), pp. 597-601.

[55] T.B. Moeslund, A. Hilton and V. Kruger, A survey of advances in vision-based human motion capture and analysis, Computer Vision and Immage Understanding 104 (2006), 90-126.

[56] I. Haritaoglu, D. Harwood and L.S. Davis, W4: real-time surveillance of people and their activities, IEEE Transactions on Pattern Analysis and Machine Intelligence 22(8) (2000), 809-830.

[57] A. Veeraraghavan, A.K. Roy-Chowdhury and R. Chellappa, Matching shape sequences in video with applications in human movement analysis, IEEE Transactions on Pattern Analysis and Machine Intelligence 27(12) (2005), 1896-1909.

[58] M. Brand, N. Oliver and A. Pentland, Coupled hidden Markov models for complex action recognition, Proceedings of the IEEE CS Conference on Computer Vision and Pattern Recognition 17-19, San Juan, Puerto Rico, (June 1997), pp. 994-999.

[59] C.T. Lin, H.W. Nein and W.C. Lin, A space-time delay neural network for motion recognition and its application to lipreading, International Journal of Neural Systems 9(4) (1999), 311-334.

[60] L. Campbell and A. Bobick, Recognition of human body motion using phase space constraints, Proceedings of the International Conference on Computer Vision 20-23,Cambridge, USA, (June 1995), pp. 624-630.

[61] J.P. Lynch and K.J. Loh, A summary review of wireless sensors and sensor networks for structural health monitoring, The Shock and Vibration Digest 38(2) (2006), 91-128.

[62] H.J. Luinge and P.H. Veltink, Measuring orientation of human body segments using miniature gyroscopes and accelerometers, Medical \& Biological Engineering \& Computing 43 (2005), 273-282.

[63] T.R. Hsu, MEMS \& Microsystems: Design and manufacture, McGraw-Hill, Boston, USA, 2002.

[64] P.H. Veltink and H.B.K. Boom, 3D movement analysis using accelerometryFtheoretical concepts, in: Europrosthetics - From Basic Research to Clinical Applications, A. Pedotti, M. Ferrarin, J. Quintern and R. Riener, eds, Springer, Berlin, Germany, 1996, pp. 317-326.

[65] R.E. Mayagoitia, A.V. Neneb and P.H. Veltink, Accelerometer and rate gyroscope measurement of kinematics: An inexpensive alternative to optical motion analysis systems, Journal of Biomechanics 35 (2002), 537-542.

[66] G. Welch and E. Foxlin, Motion tracking: No silver bullet, but a respectable arsenal, IEEE Computer Graphics and Applications 22(6) (2002), 24-38.

[67] Xsens, Xsens user manuals, Xsens Headquarters, Enschede, The Netherlands, 2011.

[68] Trivisio Prototyping GmbH, Colibri - inertial motion tracker, Trivisio Prototyping GmbH, Kaiserslautern, Germany, 2011.

[69] A.D. Young, Wireless realtime motion tracking system using localised orientation processing, PhD Thesis, University of Edinburgh, UK, 2010.

[70] J.P. Lynch, K.H. Law, A.S. Kiremidjian, T.W. Kenny, E. Carryer and A. Partridge, The design of a wireless sensing unit for structural health monitoring, Proceedings of the $3^{\text {rd }}$ International Workshop on Structural Health Monitoring, Stanford, California, USA, (September 2001), pp. 12-14. 

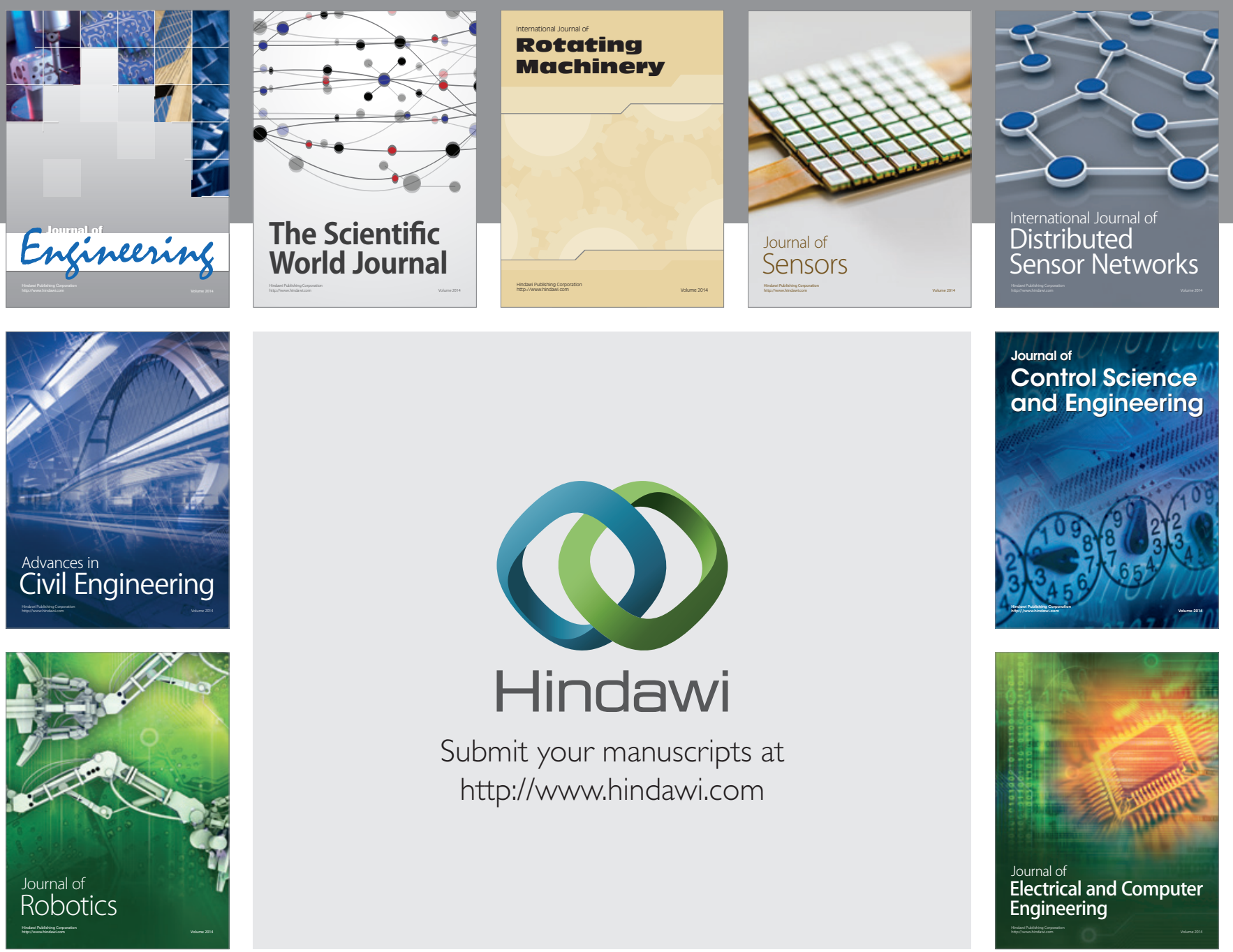

Submit your manuscripts at

http://www.hindawi.com
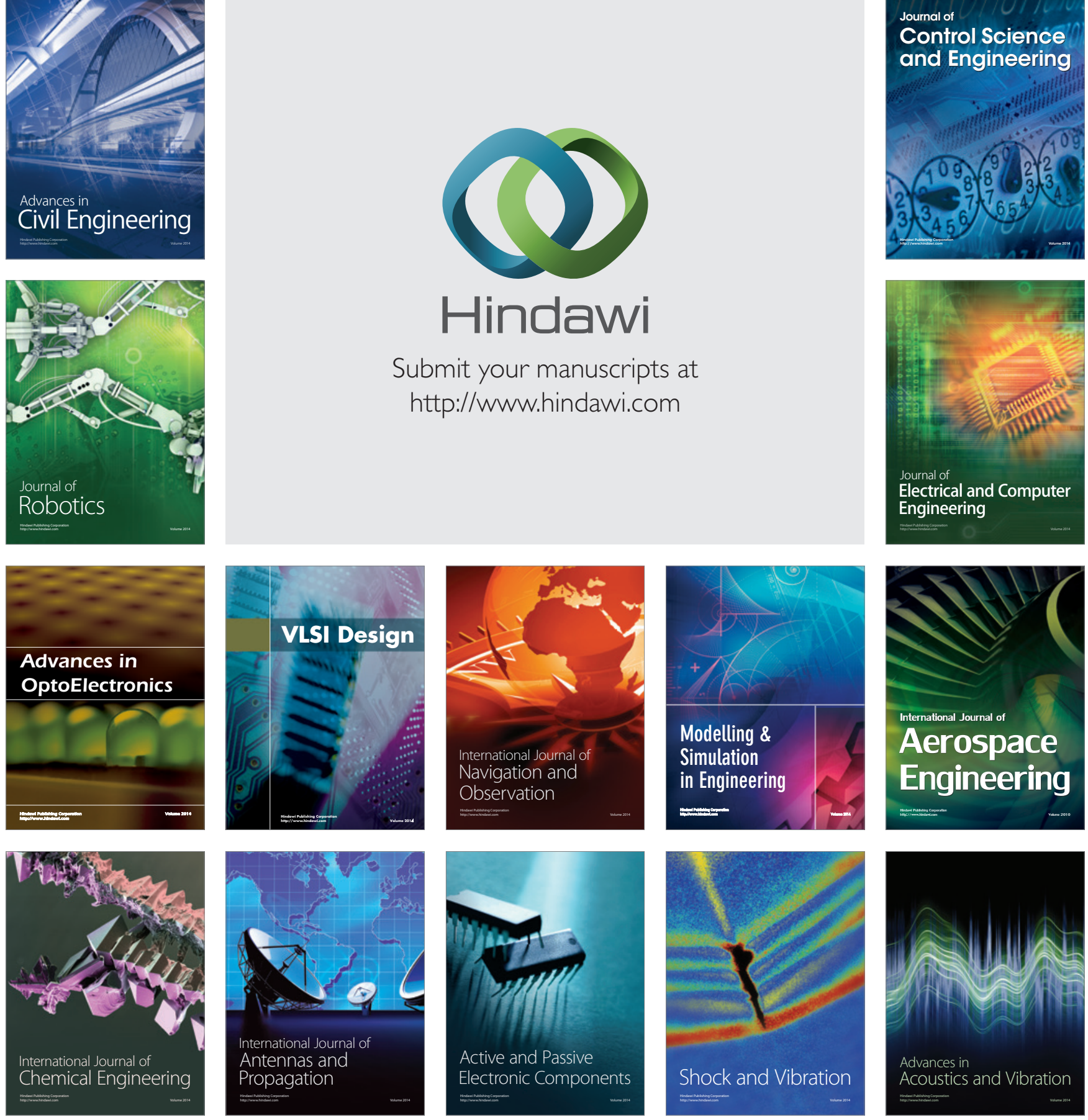